\title{
Serum microRNA signatures and metabolomics have high diagnostic value in gastric cancer
}

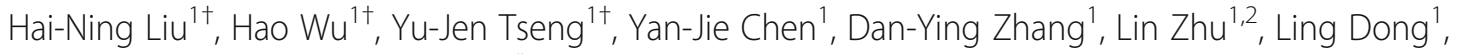
Xi-Zhong Shen ${ }^{1,3}$ and Tao-Tao Liü ${ }^{1 *}$

\begin{abstract}
Background: Many novel diagnostic biomarkers have been developed for gastric cancer (GC) recently. We chose two methods with high diagnostic value, the detection of serum microRNAs and metabolomics based on gas chromatography/mass spectrometry (GC/MS), and aimed to establish appropriate models.

Methods: We reviewed the diagnostic accuracies of all microRNAs identified by previous diagnostic tests. Then appropriate microRNAs and their combinations were validated the diagnostic value. We included 80 patients with GC and 82 healthy controls (HCs) and detected the expression of the microRNAs. GC/MS analysis was conducted, and we used three multivariate statistical analyses to establish diagnostic models. The concentrations of carcinoembryonic antigen (CEA) and carbohydrate antigen 19-9 (CA19-9) were detected for comparison with the novel models.

Results: Sixty-seven published studies and 70 microRNAs were finally included in the systematic review. MiR-18a, miR19a, miR-21, miR-92a, miR-199a and miR-421 were chosen to further validate their diagnostic efficiencies. Five of those microRNAs in GC patients had significantly different expression. The combination of miR-19a and miR-92a had the highest area under the curve (AUC) at 0.850 with a sensitivity of $91.3 \%$ and a specificity of $61.0 \%$. The GC/MS analysis performed an excellent diagnostic value and the AUC reached 1.0.

Conclusion: There is a good potential for microRNAs and GC/MS analysis as new diagnostic methods in view of their high diagnostic value compared with traditional biomarkers.
\end{abstract}

Keywords: Stomach neoplasms, Diagnostic test, Meta-analysis, microRNA, Metabolomics, Gas chromatography/mass spectrometry

\section{Background}

Gastric cancer (GC) has the fifth highest cancer morbidity and the third highest mortality rate in the world [1]. The morbidity in East Asia is much higher than that of Caucasians, and the GC patients in China are more than the sum of all other countries [2]. With the control of Helicobacter pylori infection, the changes of lifestyle, and the progress of diagnosis and treatment methods, the mortality rate of gastric cancer has gradually decreased [3, 4]. Currently, the diagnosis of GC relies

\footnotetext{
* Correspondence: liu.taotao@zs-hospital.sh.cn

${ }^{\dagger}$ Equal contributors

'Department of Gastroenterology, Zhongshan Hospital of Fudan University, Room 207, Building 3, Zhongshan Hospital, Fenglin Road 180\#, Xuhui District, Shanghai, China

Full list of author information is available at the end of the article
}

endoscopic biopsy and enhancement $\mathrm{CT}$ according to the National Comprehensive Cancer Network (NCCN) Practice Guidelines of gastric cancer. As a supplement to traditional diagnostic methods, discovering circulating biomarkers with high diagnostic value is essential. Novel diagnostic biomarkers for gastric cancer include, but are not limited to, oncogenes, tumor suppressor genes, microRNAs and long non-coding RNAs, DNA methylation and low-molecular-weight metabolites [5-7]. Considering the diagnostic accuracies, advantages and disadvantages, we chose two methods, the detection of serum microRNAs and metabolomics based on gas chromatography/mass spectrometry (GC/MS), to validate their diagnostic efficiencies and attempt to develop appropriate models.

(c) The Author(s). 2018 Open Access This article is distributed under the terms of the Creative Commons Attribution 4.0 International License (http://creativecommons.org/licenses/by/4.0/), which permits unrestricted use, distribution, and reproduction in any medium, provided you give appropriate credit to the original author(s) and the source, provide a link to the Creative Commons license, and indicate if changes were made. The Creative Commons Public Domain Dedication waiver (http://creativecommons.org/publicdomain/zero/1.0/) applies to the data made available in this article, unless otherwise stated. 
MicroRNAs are non-protein-coding RNAs with small molecular size that regulate target gene expression by binding to their 3' untranslated region [8]. Thousands of microRNAs have been discovered over the past decade, and quite a few microRNAs have been determined the potential for the diagnosis of GC. Nevertheless, the diagnostic efficiencies of the reported circulating microRNAs are not consistent among studies. It is thus necessary to summarize the diagnostic value of these microRNAs via a systematic review. We did abovementioned work and aimed to overcome the deficiencies of previous systematic reviews and meta-analyses, such as small including article number, single researched microRNA [9], or lack of the information of each microRNA [10-13]. Then we chose six microRNAs with high Youden indexes or area under the curve (AUC) values of the receiver operating curve (ROC) to validate their diagnostic value and establish a diagnostic panel.

Metabolomics is defined as the quantitative measurement of low-molecular-weight metabolites in an organism at a specified time under specific environmental conditions [14]. GC/MS, which is one of metabolomic techniques, has robust results and is widely used in metabolite identification because of its peak resolution, high sensitivity, and reproducibility $[15,16]$. Several studies reported its high diagnostic value for GC, and the AUC value usually reached more than 0.90 [17]. As highthroughput experimental data, the results of GC/MS are always processed by multivariate statistical analysis, including the principal component analysis (PCA), partial least squares-discriminate analysis (PLS-DA), and orthogonal partial least squares-discriminant analysis (OPLS-DA). We further validated the diagnostic value of metabolomics and compared the three most frequently used statistical methods.

\section{Methods \\ Study design}

First of all, we reviewed the diagnostic accuracies of microRNAs mentioned in previous studies. We searched several relevant databases, including PubMed, Embase, and the Chinese Biomedical Literature Database (CBM) up to Jul 26, 2017. The search strategy was ("stomach neoplasms"[Mesh] OR "gastric cancer" OR "stomach cancer") AND (miRNA OR microRNA OR miR) AND (blood OR serum OR plasma OR circulating) AND (diagnosis OR diagnostic OR diagnose). There were no language restrictions in searching process. Lists of references of articles were searched manually for additional publications [18].

Then, we selected the microRNAs with high Youden indexes and high AUC values to establish a diagnostic model according to the results of the systematic review. The serum specimens from 80 patients with GC and 82 healthy controls ( $\mathrm{HCs}$ ) were obtained to detect the microRNA levels using quantitative reverse-transcription polymerase chain reaction (qRT-PCR).

Next, we selected 25 GC patients and $30 \mathrm{HCs}$ from the cohort mentioned above with a completely random method and utilized GC/MS to profile the metabolomic signatures.

Finally, the diagnostic value was compared among the new models and the traditional tumor biomarkers, carcinoembryonic antigen (CEA) and carbohydrate antigen 19-9 (CA19-9). An overview of the study design is illustrated in Fig. 1.

\section{Inclusion and exclusion criteria of the literature}

Studies were included if they met the following inclusion criteria: (1) studies regarding the diagnostic value of microRNAs in GC; (2) blood specimens; and (3) qRTPCR techniques. Additionally, studies exclusion criteria are: (1) failure to provide enough diagnostic information; (2) duplicate publications reported by identical authorities; and (3) animal or cell studies, letters and reviews.

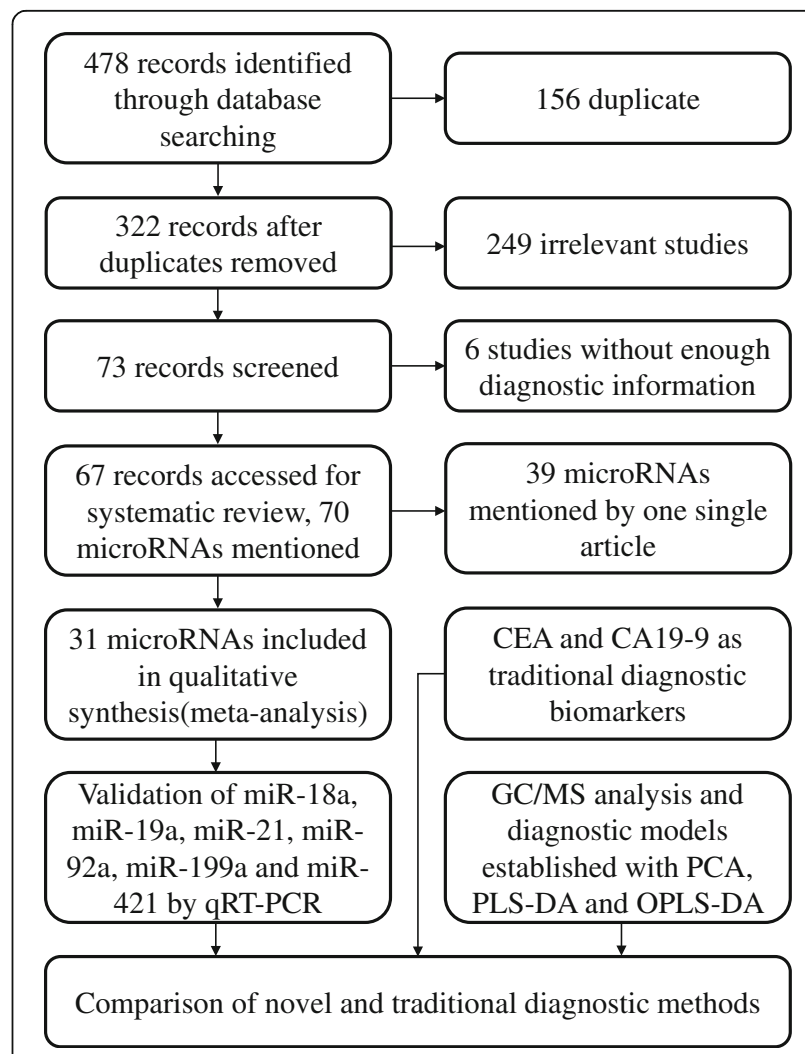

Fig. 1 Flow diagram of trial selection. Abbreviations: CEA, carcinoembryonic antigen; CA19-9, carbohydrate antigen 19-9; qRT-PCR quantitative reverse-transcription polymerase chain reaction; GC/MS, gas chromatography/mass spectrometry; PCA, principal component analysis; PLS-DA, partial least squares-discriminate analysis; OPLS-DA, orthogonal partial least squares-discriminant analysis 


\section{Data extraction}

Data were extracted independently by two reviewers from all of the included articles: (1) basic characteristics of the studies, including the first author, year of publication, country of publication, ethnicity, sample size, mean or median age, gender, type of specimens (serum or plasma), target microRNAs, and reference control RNA; and (2) diagnostic information of the microRNAs, including the sensitivity, specificity, AUC and expression variation.

\section{Patients and specimens}

We included 80 patients with GC and $82 \mathrm{HCs}$ who were from in Zhongshan Hospital, Fudan University between May 2015 and September 2015. The GC patients were all definitively diagnosed by an endoscopic biopsy. Exclusion criteria were history of other malignant tumors, a surgical operation, radiotherapy or chemotherapy. Healthy individuals were identified by clinical manifestations, histories of diseases and results of blood tests. The samples were centrifuged for $10 \mathrm{~min}$ at $820 \mathrm{~g}$ and $4{ }^{\circ} \mathrm{C}$ to remove residual cell debris, and the supernatants were immediately stored at $-80{ }^{\circ} \mathrm{C}$ until further analyses. The serum concentrations of serum CEA and CA19-9 were measured with the electro-chemiluminescence immunoassay.

Approval for the study was given by the Ethics Committee of Zhongshan Hospital of Fudan University, Shanghai. All GC patients and control subjects provided written informed consents before enrollment in this study.

\section{RNA extraction and reverse transcription}

$200 \mu \mathrm{l}$ of the serum samples was spiked with $2 \mu \mathrm{l}$ of 25 fmol synthetic cel-miR-39 (Tiangen, Beijing, China) as the external reference. Total RNA enriched for small RNAs was isolated simultaneously from the serum with the miRcute microRNA Isolation Kit (Tiangen, Beijing, China) according to the modified manufacturer's protocol [19]. To determine the purities and concentrations, we utilized a NanoDrop spectrophotometer (NanoDrop, Wilmington, DE, USA) to assess the optical density of the extracted RNA at 260 and $280 \mathrm{~nm}$.

The extracted microRNA was polyadenylated by $20 \mu \mathrm{l}$ of the poly (A) polymerase. $6 \mu$ l of the poly (A) reaction solution was reverse transcribed to cDNA in another $20 \mu \mathrm{l}$ with miRcute microRNA The First-strand cDNA Synthesis Kit (Tiangen, Beijing, China) following the manufacturer's instructions. Reverse transcription was run in triplicate.

\section{Quantitative real-time PCR}

The PCR reaction was performed for amplification using the miRcute microRNA qPCR Detection Kit (Tiangen, Beijing, China) on ABI PRISM 7500 Sequence Detection
System (Applied Biosystems, Foster City, CA, USA). Each qPCR reaction solution contained diluted cDNA, $2 \times$ miRcute microRNA premix (with SYBR and ROX), the manufacturer-provided microRNA-specific forward primer, and a universal reverse primer to a total volume of $20 \mu \mathrm{l}$. The qPCR reaction parameters were $94{ }^{\circ} \mathrm{C}$ predenaturation for $2 \mathrm{~min}, 45$ cycles of $94{ }^{\circ} \mathrm{C}$ for $20 \mathrm{~s}, 60{ }^{\circ} \mathrm{C}$ annealing for $34 \mathrm{~s}$, and $72{ }^{\circ} \mathrm{C}$ extension for $30 \mathrm{~s}$. A melting curve analysis was accomplished to ensure the specificity of the target PCR product in the end.

The relative expression of the microRNAs was calculated using the equation $\log _{10}\left(2^{-\Delta C T}\right)$. The $\Delta C T$ was equal to CT values of the microRNAs of interest minus the CT values of the cel-miR-39 [19].

\section{Specimen processing for metabolomics}

For the GC/MS analysis, the serum samples were transferred into glass centrifuge tubes in a $200-\mu \mathrm{l}$ volume. Each sample was spiked with $200 \mu \mathrm{l}$ of 2-chloro-phenylalanine $(0.3 \mathrm{~g} / \mathrm{L})$ as an internal standard and $600 \mu \mathrm{l}$ of methanol. The mixture was vortexed for $30 \mathrm{~s}$, incubated for $10 \mathrm{~min}$ at $-20{ }^{\circ} \mathrm{C}$ and then centrifuged for $15 \mathrm{~min}$ at $12000 \times \mathrm{g}$ and $4{ }^{\circ} \mathrm{C}$. Supernatant in an $800-\mu \mathrm{l}$ volume was collected separately into an ampoule bottle and then evaporated to dryness under a stream of nitrogen gas at $50{ }^{\circ} \mathrm{C}$ for around $30 \mathrm{~min}$. Subsequently, $200 \mu \mathrm{l}$ of a methoxyamine pyridine solution $(15 \mathrm{~g} / \mathrm{L})$ was put into the ampoule bottle. The mixture was vortexed for $2 \mathrm{~min}$ and incubated for $60 \mathrm{~min}$ at $37{ }^{\circ} \mathrm{C}$. Next, we added $200 \mu \mathrm{l}$ of bis-(trimethylsilyl)-trifluoroacetamide (BSTFA) plus $1 \%$ trimethylchlorosilane (TMCS), and the mixture was vortexed for $2 \mathrm{~min}$ and incubated for $30 \mathrm{~min}$ at $100{ }^{\circ} \mathrm{C}$. The methanol, 2-chlorophenylalanine, methoxyamine and pyridine were bought from Aladdin (Shanghai, China). The BSTFA with 1\% TMCS was bought from Sigma-Aldrich (St. Louis, MO, USA). All reaction samples were performed in duplicate.

\section{GC/MS analysis}

The GC/MS analysis was carried out on an Agilent 6980 GC system equipped with a fused-silica capillary column with a $0.25-\mu \mathrm{m}$ HP-5MS stationary phase (Agilent, Shanghai, China). We used the same operational methods as our previous studies [20].

\section{Statistical analyses}

The statistical analyses were conducted with Stata 12.0 (StataCorp LP, College Station, TX, USA), SIMCA-P 13. 0 (Umetrics AB, Umea, Vasterbotten, Sweden) and $R$ software 3.3.3 (R Foundation for Statistical Computing, Vienna, Austria). A $P$ value less than 0.05 was considered statistically significant.

Meta-analysis methods for diagnostic tests were used to assess the value of the individual microRNAs to diagnose GC using the sensitivity, specificity and AUC of the 
Table 1 Characteristics of the microRNAs mentioned in the literature

\begin{tabular}{|c|c|c|c|c|c|c|c|}
\hline MicroRNA & Expression & GC sample size & Control sample size & Sensitivity (\%) & Specificity (\%) & AUC & $\begin{array}{l}\text { Number of } \\
\text { included articles }\end{array}$ \\
\hline miR-451 & $U$ & 144 & 172 & 91.2 & 96.5 & 0.980 & 2 \\
\hline miR-183 & $U$ & 76 & 26 & 93.4 & 92.3 & 0.978 & 1 \\
\hline miR-300 & $U$ & 25 & 15 & 96.0 & 86.7 & NA & 1 \\
\hline miR-940 & $\mathrm{D}$ & 110 & 100 & 73.2 & 97.9 & 0.970 & 1 \\
\hline miR-486 & $U$ & 144 & 172 & 78.1 & 94.0 & 0.950 & 2 \\
\hline miR-421 & $U$ & 130 & 107 & 92.3 & 79.4 & 0.940 & 2 \\
\hline miR-100 & $U$ & 90 & 87 & 86.9 & 87.3 & 0.930 & 2 \\
\hline miR-19a & $U$ & 215 & 241 & 83.1 & 89.5 & 0.930 & 3 \\
\hline miR-19b & $U$ & 215 & 241 & 76.6 & 88.4 & 0.910 & 3 \\
\hline miR-627 & $U$ & 108 & 96 & 80.0 & 89.6 & 0.900 & 1 \\
\hline miR-21 & $U \& D$ & 334 & 250 & 77.8 & 87.3 & 0.900 & 6 \\
\hline miR-204 & $\mathrm{D}$ & 94 & 100 & 73.4 & 82.6 & 0.896 & 1 \\
\hline miR-206 & $D$ & 150 & 150 & 78.0 & 86.0 & 0.890 & 1 \\
\hline miR-200c & $U \& D$ & 233 & 145 & 81.8 & 81.8 & 0.890 & 4 \\
\hline miR-503 & $\mathrm{D}$ & 68 & 32 & 96.8 & 79.4 & 0.889 & 1 \\
\hline miR-26a & $\mathrm{D}$ & 280 & 280 & 83.6 & 81.5 & 0.882 & 1 \\
\hline miR-17-3p & U & 154 & 180 & 69.7 & 88.9 & 0.880 & 2 \\
\hline miR-652 & U & 108 & 96 & 65.7 & 88.8 & 0.880 & 1 \\
\hline miR-130a & U & 41 & 41 & 77.9 & 90.2 & 0.870 & 1 \\
\hline miR-629 & U & 108 & 96 & 85.2 & 82.0 & 0.870 & 1 \\
\hline miR-196a & U & 98 & 126 & 69.5 & 97.6 & 0.864 & 1 \\
\hline miR-378 & $U$ & 40 & 41 & 87.5 & 70.7 & 0.861 & 1 \\
\hline miR-101 & U & 58 & 60 & 71.0 & 88.1 & 0.857 & 1 \\
\hline miR-233 & U & 50 & 47 & 81.0 & 78.0 & 0.850 & 1 \\
\hline miR-106b & U & 314 & 275 & 72.0 & 84.3 & 0.850 & 4 \\
\hline miR-142-3p & $\mathrm{D}$ & 280 & 280 & 74.4 & 84.1 & 0.839 & 1 \\
\hline miR-192 & U & 29 & 10 & 75.9 & 90.0 & 0.833 & 1 \\
\hline miR-18a & U & 500 & 439 & 73.2 & 80.8 & 0.830 & 6 \\
\hline miR-199a & U & 415 & 296 & 78.1 & 74.3 & 0.830 & 4 \\
\hline miR-6503-5p & U & 361 & 103 & 57.9 & 88.3 & 0.830 & 2 \\
\hline miR-200b & $\mathrm{D}$ & 84 & 30 & 62.7 & 93.4 & 0.826 & 1 \\
\hline miR-92a & U & 382 & 665 & 75.0 & 77.6 & 0.820 & 5 \\
\hline miR-20a & U & 615 & 557 & 71.8 & 77.8 & 0.820 & 6 \\
\hline miR-223 & U & 211 & 211 & 70.8 & 78.7 & 0.820 & 4 \\
\hline miR-196b & U & 98 & 126 & 62.2 & 96.1 & 0.811 & 1 \\
\hline miR-16 & U & 138 & 189 & 76.6 & 87.6 & 0.810 & 2 \\
\hline miR-25 & U & 634 & 431 & 67.6 & 83.5 & 0.810 & 5 \\
\hline miR-1 & U & 240 & 166 & 79.2 & 73.2 & 0.800 & 2 \\
\hline miR-375 & $\mathrm{D}$ & 91 & 91 & 75.3 & 73.6 & 0.800 & 3 \\
\hline miR-140-5p & U & 108 & 96 & 81.7 & 70.0 & 0.790 & 1 \\
\hline miR-106a & $U \& D$ & 287 & 159 & 70.0 & 79.7 & 0.790 & 4 \\
\hline miR-744 & U & 287 & 228 & 75.7 & 72.0 & 0.790 & 2 \\
\hline miR-210 & U & 201 & 191 & 76.8 & 63.9 & 0.780 & 2 \\
\hline
\end{tabular}


Table 1 Characteristics of the microRNAs mentioned in the literature (Continued)

\begin{tabular}{|c|c|c|c|c|c|c|c|}
\hline MicroRNA & Expression & GC sample size & Control sample size & Sensitivity (\%) & Specificity (\%) & AUC & $\begin{array}{l}\text { Number of } \\
\text { included articles }\end{array}$ \\
\hline miR-181b & $U$ & 46 & 21 & 77.2 & 72.7 & 0.770 & 1 \\
\hline miR-27a & $U$ & 149 & 124 & 71.8 & 70.8 & 0.770 & 3 \\
\hline miR-17-5p & $U \& D$ & 313 & 237 & 55.3 & 80.4 & 0.770 & 4 \\
\hline miR-195 & $\mathrm{D}$ & 280 & 280 & 69.2 & 75.4 & 0.765 & 1 \\
\hline miR-222 & U & 182 & 124 & 62.3 & 82.6 & 0.760 & 2 \\
\hline miR-148a & $\mathrm{D}$ & 331 & 331 & 60.2 & 76.2 & 0.760 & 2 \\
\hline miR-93 & U & 65 & 65 & 81.5 & 73.8 & 0.756 & 1 \\
\hline miR-218 & $\mathrm{D}$ & 60 & 60 & 94.3 & 44.3 & 0.743 & 1 \\
\hline miR-23b & $U \& D$ & 184 & 104 & 71.3 & 88.9 & 0.730 & 2 \\
\hline miR-191 & U & 125 & 126 & 61.8 & 97.6 & 0.730 & 2 \\
\hline miR-215 & U & 29 & 10 & 62.1 & 88.3 & 0.724 & 1 \\
\hline miR-371-5p & U & 40 & 41 & 75.0 & 63.4 & 0.715 & 1 \\
\hline miR-376c & U & 68 & 68 & 74.0 & 62.7 & 0.710 & 1 \\
\hline miR-187* & U & 40 & 41 & 82.5 & 61.0 & 0.704 & 1 \\
\hline let-7e & U & 68 & 68 & 62.1 & 79.2 & 0.700 & 1 \\
\hline miR-320a & D & 35 & 38 & 65.2 & 68.2 & 0.699 & 1 \\
\hline miR-92b & U & 101 & 91 & 46.9 & 88.2 & 0.690 & 1 \\
\hline miR-221 & U & 248 & 248 & 63.1 & 92.1 & 0.670 & 3 \\
\hline miR-27b & U & 68 & 68 & 48.7 & 82.0 & 0.660 & 1 \\
\hline miR-107 & $U \& N$ & 86 & 86 & 40.7 & 80.2 & 0.660 & 2 \\
\hline miR-185 & U & 101 & 91 & 46.7 & 84.9 & 0.650 & 1 \\
\hline miR-34a & U & 180 & 106 & 59.4 & 67.8 & 0.645 & 1 \\
\hline miR-151-5p & U & 180 & 80 & 61.0 & 57.0 & 0.625 & 1 \\
\hline miR-423-5p & U & 180 & 106 & 87.3 & 31.6 & 0.590 & 1 \\
\hline miR-103 & $\mathrm{N}$ & 50 & 50 & 93.6 & 24.4 & 0.548 & 1 \\
\hline miR-425 & U & 57 & 58 & 31.7 & 84.2 & 0.548 & 1 \\
\hline miR-194 & $\mathrm{N}$ & 50 & 50 & 94.2 & 24.4 & 0.512 & 1 \\
\hline
\end{tabular}

We use $\mathrm{U}$ to represent the upregulated expression, use $\mathrm{D}$ to represent the downregulated expression and use $\mathrm{N}$ to represent no significant difference in the GC patients versus the control group. The data on the sensitivity, specificity and AUC were obtained via the meta-analysis when the number of included articles was more than one

Abbreviations: GC gastric cancer, AUC area under the curve, NA not available

summary receiver operator characteristic (SROC). Deeks' funnel plot was adopted to evaluate the publication bias.

A power analysis was used to obtain the sample size of the GC cases and controls in the microRNA validation phase. Wilcoxon-Mann-Whitney test and Student's $t$-test were used for the comparison between the patients and the HCs, including the expression of the microRNAs and the concentrations of CEA and CA19-9. The diagnostic efficiencies of the microRNAs were assessed with the sensitivity, specificity and the AUC of the ROC. A logistic regression was utilized to build an appropriate diagnostic model.

The metabolomic information was normalized with "XCMS" package in R software and the data were edited into a two-dimensional matrix, including the mass-tocharge ratio (MZ), retention time (RT) and peak intensity. SIMCA-P software was used to perform multivariate data analyses, including PCA, PLS-DA, and OPLS-DA. A logistic regression was used to investigate the better diagnostic model by combinations of the various components when more than one component was extracted. The metabolites were identified based on the National Institute of Standards and Technology (NIST) mass spectra library through RT and MZ [20]. We screened the significantly different metabolites via the variable importance in the projection (VIP) value $(>1)$ of the OPLS-DA model and the $P$ value $(<0.001)$ of fold change of Student's $t$-test between the patients and the HCs. 
Table 2 Clinical and pathological characteristics of the study population

\begin{tabular}{|c|c|c|c|}
\hline Variable & Patients $(n=80)$ & Control subjects $(n=82)$ & $P$ value \\
\hline Age (year) & $65.1 \pm 10.5$ & $34.8 \pm 7.3$ & $<0.001$ \\
\hline \multicolumn{3}{|l|}{ Gender } & 0.090 \\
\hline Male & 57 & 48 & \\
\hline Female & 23 & 34 & \\
\hline Tumor size $(\mathrm{cm})$ & $4.27 \pm 2.62$ & & \\
\hline$>5$ & 49 & & \\
\hline$\geq 5$ & 31 & & \\
\hline \multicolumn{4}{|l|}{ TNM stage } \\
\hline । & 20 & & \\
\hline$\|$ & 18 & & \\
\hline$\|$ & 31 & & \\
\hline IV & 11 & & \\
\hline \multicolumn{4}{|l|}{ Histological grade } \\
\hline I & 4 & & \\
\hline$\|$ & 14 & & \\
\hline$\|\sim\|$ & 25 & & \\
\hline IV & 37 & & \\
\hline \multicolumn{4}{|c|}{ Lauren classification } \\
\hline Diffuse & 25 & & \\
\hline Intestinal & 31 & & \\
\hline Mixed & 24 & & \\
\hline \multicolumn{4}{|c|}{ Tumor localization } \\
\hline Cardia & 19 & & \\
\hline Corpus & 8 & & \\
\hline Antrum & 43 & & \\
\hline Whole & 10 & & \\
\hline
\end{tabular}

\section{Results}

Study selection and literature characteristics

The initial search returned a total of 478 records, among which, 146 were from PubMed, 249 were from Embase, and 83 were from CBM. We removed 156 duplicates, 249 irrelevant studies and six articles that failed to provide enough diagnostic information. Sixty-seven candidate articles were finally enrolled into this systematic review with a total of $5261 \mathrm{GC}$ patients and 4386 healthy controls (Additional file 1: Table S1 and Additional file 2: Table S2).

\section{Diagnostic value of microRNAs in the literature}

There were 70 microRNAs mentioned in the included articles, of which, 39 were studied in one single article. We performed the meta-analyses to represent the diagnostic value of the other 31 microRNAs. The details regarding each microRNA are displayed in Table 1.

\section{Publication bias}

Publication bias was assessed with a Deeks' funnel plot (Additional file 3: Figure S1), and the $P$ value of Deeks' test was 0.24 . Therefore, there was no evidence showing that publication bias existed.

\section{Study population}

The clinical and pathological features of the patients and HCs are presented in Table 2. Age was found significant differences between the GC patients and the HCs. We thus performed a covariance analysis. The results suggested that there were no correlations between age and either the expression of the microRNAs, the scores of

Abbreviation: TNM tumor-node-metastasis

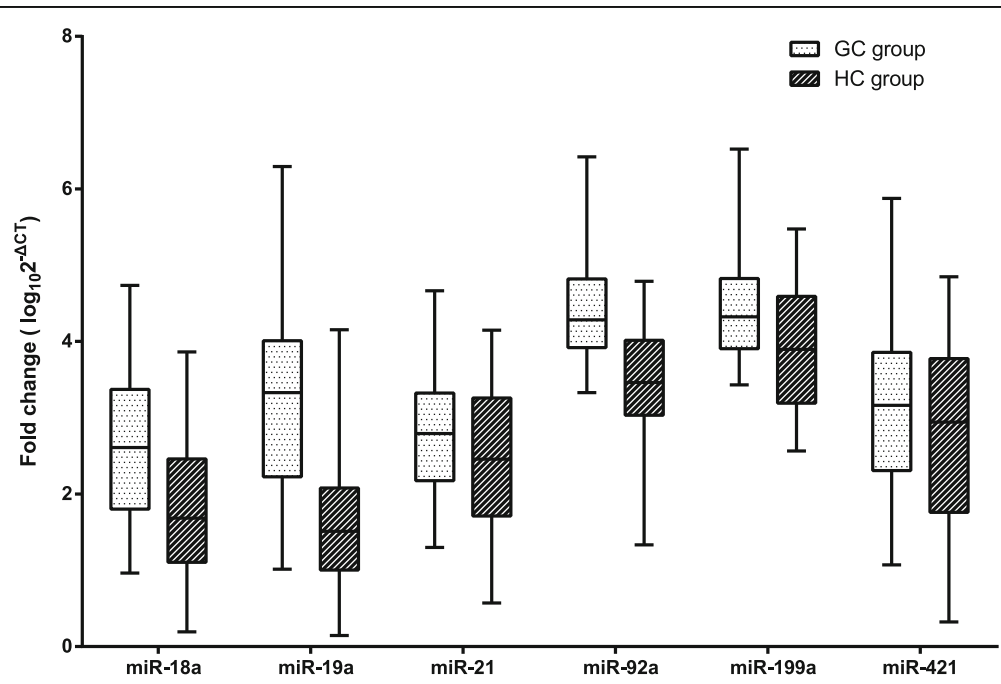

Fig. 2 Box plots for the expression of the seven microRNAs. The $P$ values of miR-18a, miR-19a, miR-21, miR-92a, miR-199a and miR-421 were $<0.001,<0.001$, $0.024,<0.001,<0.001$ and 0.098 , respectively. The lines within the boxes represent the median values, and the edges of the boxes demonstrate the interquartile ranges. The lines outside the boxes demonstrate the 95\% ranges. Abbreviations: GC, gastric cancer; HC, healthy control 
the components of the metabolomics or the concentrations of CEA and CA19-9.

\section{Expression of microRNAs}

MiR-18a, miR-19a, miR-21, miR-92a, miR-199a and miR-421 were chosen in view of their high diagnostic efficiencies in previous studies. The results of the qRTPCR showed that the serum levels of the microRNAs except miR-421 in the GC patients were significantly higher than those in the HCs (Additional file 4: Table S3 and Fig. 2). The expression of miR-421 wasn't observed significant difference between the patients and HCs.

\section{Diagnostic models established using microRNAs}

We calculated the sensitivity, specificity, AUC value of each microRNA and their combinations at the optimal cut-off value to find the appropriate diagnostic model (Table 3). The combination of miR-19a and miR-92a had the highest AUC value at 0.850 , with a sensitivity of 91 . $3 \%$ and a specificity of $61.0 \%$. The cut-off value of the

Table 3 Diagnostic value of five single microRNAs and their combinations

\begin{tabular}{|c|c|c|c|c|c|}
\hline MicroRNA(s) & Sensitivity (\%) & Specificity (\%) & AUC (95\% Cl) & Cut-off value & $\overline{\text { Youden index }}$ \\
\hline miR-18a & 65.0 & 69.5 & $0.731(0.655,0.807)$ & 2.201 & 0.345 \\
\hline miR-19a & 80.0 & 75.6 & $0.821(0.757,0.885)$ & 2.072 & 0.556 \\
\hline miR-21 & 77.5 & 42.7 & $0.590(0.502,0.678)$ & 2.133 & 0.202 \\
\hline miR-92a & 95.0 & 56.1 & $0.817(0.753,0.881)$ & 3.533 & 0.511 \\
\hline miR-199a & 97.5 & 36.6 & $0.684(0.602,0.766)$ & 3.530 & 0.341 \\
\hline miR-421 & 93.8 & 20.7 & $0.556(0.467,0.644)$ & 1.525 & 0.145 \\
\hline miR-18a + miR-19a & 76.3 & 80.5 & $0.833(0.772,0.894)$ & 3.600 & 0.567 \\
\hline$m i R-18 a+m i R-21$ & 55.0 & 80.5 & $0.729(0.652,0.806)$ & 2.454 & 0.355 \\
\hline$m i R-18 a+m i R-92 a$ & 96.3 & 58.5 & $0.823(0.760,0.885)$ & 6.901 & 0.548 \\
\hline miR-18a + miR-199a & 78.8 & 58.5 & $0.746(0.672,0.820)$ & 3.865 & 0.373 \\
\hline$m i R-19 a+m i R-21$ & 78.8 & 75.6 & $0.820(0.756,0.884)$ & 2.086 & 0.544 \\
\hline $\operatorname{miR}-19 a+m i R-92 a$ & 91.3 & 61.0 & $0.850(0.794,0.907)$ & 6.898 & 0.522 \\
\hline miR-19a + miR-199a & 80.0 & 72.0 & $0.819(0.755,0.883)$ & 3.580 & 0.520 \\
\hline$m i R-21+m i R-92 a$ & 91.3 & 63.4 & $0.820(0.757,0.884)$ & 7.300 & 0.547 \\
\hline$m i R-21+m i R-199 a$ & 93.8 & 36.6 & $0.681(0.600,0.762)$ & 3.661 & 0.303 \\
\hline miR-92a + miR-199a & 81.3 & 70.7 & $0.818(0.754,0.881)$ & 7.272 & 0.520 \\
\hline miR-18a + miR-19a + miR-21 & 75.0 & 81.7 & $0.835(0.774,0.896)$ & 3.323 & 0.567 \\
\hline miR-18a + miR-19a + miR-92a & 72.5 & 85.4 & $0.857(0.801,0.912)$ & 7.780 & 0.579 \\
\hline miR-18a + miR-19a + miR-199a & 78.8 & 76.8 & $0.836(0.775,0.896)$ & 4.025 & 0.556 \\
\hline$m i R-18 a+m i R-21+m i R-92 a$ & 93.8 & 62.2 & $0.828(0.766,0.890)$ & 6.884 & 0.559 \\
\hline$m i R-18 a+m i R-21+m i R-199 a$ & 81.3 & 57.3 & $0.746(0.672,0.820)$ & 3.845 & 0.386 \\
\hline$m i R-18 a+m i R-92 a+m i R-199 a$ & 98.8 & 52.4 & $0.826(0.764,0.888)$ & 6.174 & 0.512 \\
\hline$m i R-19 a+m i R-21+m i R-92 a$ & 81.3 & 72.0 & $0.859(0.805,0.913)$ & 7.140 & 0.532 \\
\hline miR-19a + miR-21 + miR-199a & 80.0 & 72.0 & $0.820(0.756,0.883)$ & 3.410 & 0.520 \\
\hline$m i R-19 a+m i R-92 a+m i R-199 a$ & 72.5 & 81.7 & $0.857(0.802,0.912)$ & 7.188 & 0.542 \\
\hline miR-21 + miR-92a + miR-199a & 92.5 & 62.2 & $0.820(0.757,0.884)$ & 6.830 & 0.547 \\
\hline$m i R-18 a+m i R-19 a+m i R-21+m i R-92 a$ & 82.5 & 73.2 & $0.861(0.807,0.915)$ & 6.938 & 0.557 \\
\hline$m i R-18 a+m i R-19 a+m i R-21+m i R-199 a$ & 75.0 & 80.5 & $0.836(0.775,0.897)$ & 4.022 & 0.555 \\
\hline$m i R-18 a+m i R-19 a+m i R-92 a+m i R-199 a$ & 77.5 & 79.3 & $0.861(0.806,0.915)$ & 6.876 & 0.568 \\
\hline$m i R-18 a+m i R-21+m i R-92 a+m i R-199 a$ & 97.5 & 58.5 & $0.830(0.768,0.891)$ & 6.166 & 0.560 \\
\hline$m i R-19 a+m i R-21+m i R-92 a+m i R-199 a$ & 80.0 & 75.6 & $0.862(0.808,0.915)$ & 6.491 & 0.556 \\
\hline miR-18a + miR-19a + miR-21 + miR-92a + miR-199a & 87.5 & 69.5 & $0.867(0.814,0.920)$ & 5.983 & 0.570 \\
\hline
\end{tabular}

The bold font indicates that the $P$ value of every microRNA in the combination was less than 0.05 in the logistic regression Abbreviation: $A \cup C$ area under the curve, $\mathrm{Cl}$ confidence interval 
model was 6.90 , according to the formula miR-19a $\times 0$. $750+$ miR-92a $\times 1.455$.

\section{Discrepant metabolites and total ion chromatogram}

A total of 1118 features were extracted in GC/MS analysis. We found 25 significantly different metabolites (Additional file 5: Table S4). The retention time in the total ion chromatograms was stable with no drift in all of the peaks, which implied that the results were credible.

\section{Diagnostic models established using metabolomics}

We extracted eleven principal components in the PCA model, while eigenvalues in seven of the eleven principal components were more than 1.0. We calculated the diagnostic efficiencies when fitting into one to eleven principal components. When enrolled into more than six principal components, the AUC value reached up to 1.0. Five components were extracted in the PLS-DA model, and the AUC values were all higher than those in the PCA model with the same number of components. Just one factor was extracted in the OPLS-DA model, and the AUC value was 1.0.

More details of diagnostic information from the three statistical methods are presented in Table 4 and Fig. 3.

\section{Diagnostic value of traditional tumor biomarkers}

The CEA concentration in GC patients was significantly higher than that of HCs (Wilcoxon-Mann-Whitney test,
$P<0.001)$. The median concentrations in the patients and HCs were 2.6 (range, 0.5-302.4) and 1.3 (range, 0. 3-4.2) $\mu \mathrm{g} / \mathrm{L}$, respectively. For CEA, the sensitivity was $45.0 \%$ and the specificity was $95.1 \%$ with an AUC of 0 . 763 (95\% CI $=0.686-0.839)$ when the cut-off value was $2.85 \mu \mathrm{g} / \mathrm{L}$. When the cut-off value was set at $5 \mu \mathrm{g} / \mathrm{L}$, which is the traditional upper bound of healthy people, the sensitivity was $22.5 \%$, and the specificity was $100 \%$.

The CA19-9 concentration wasn't showed significant difference between GC patients and HCs (WilcoxonMann-Whitney test, $P=0.203$ ). The median concentrations in the patients and HCs were 9.0 (range, 0.6-423. 6) and 7.3 (range, $0.6-26.8) \mathrm{U} / \mathrm{ml}$, respectively. The AUC of CA19-9 was 0.563 (95\% CI, 0.468-0.657; sensitivity $=71.3 \%$, specificity $=44.3 \%)$ at the cut-off value of $6.75 \mathrm{U} / \mathrm{ml}$. When the cut-off value was at $37 \mathrm{U} / \mathrm{ml}$, the sensitivity was $12.5 \%$, and the specificity was $100 \%$.

The ROC curves of the new models and the traditional tumor biomarkers are displayed in Fig. 4.

\section{Discussion}

The development of new technologies has spawned a series of new diagnostic biomarkers. Genomics, microarrays, proteomics, and metabolomics have become general methods for finding novel biomarkers [5]. After reviewing the oncogenes (MMP-9, STC1 and S100A6) [21-23], DNA methylated markers (APBA2, SPG20 and SOX17) [24-26], IncRNAs (UCA1 and LSINCT-5) [27] and the combinations of autoantibody spectrum $[28,29]$,

Table 4 Diagnostic value of the gas chromatography/mass spectrometry analysis with multivariate statistical analysis methods

\begin{tabular}{|c|c|c|c|c|c|c|}
\hline Statistical method & Number of components & Sensitivity (\%) & Specificity (\%) & AUC (95\% Cl) & Youden index & Cumulative variance \\
\hline \multirow[t]{11}{*}{ PCA } & 11 & 100.0 & 100.0 & $1.000(1.000,1.000)$ & 1.000 & 0.445 \\
\hline & 10 & 100.0 & 100.0 & $1.000(1.000,1.000)$ & 1.000 & 0.379 \\
\hline & 9 & 100.0 & 100.0 & $1.000(1.000,1.000)$ & 1.000 & 0.195 \\
\hline & 8 & 100.0 & 100.0 & $1.000(1.000,1.000)$ & 1.000 & 0.153 \\
\hline & 7 & 100.0 & 100.0 & $1.000(1.000,1.000)$ & 1.000 & 0.127 \\
\hline & 6 & 92.0 & 96.7 & $0.984(0.960,1.000)$ & 0.887 & 0.120 \\
\hline & 5 & 92.0 & 93.3 & $0.980(0.952,1.000)$ & 0.853 & 0.086 \\
\hline & 4 & 88.0 & 96.7 & $0.976(0.945,1.000)$ & 0.847 & 0.070 \\
\hline & 3 & 92.0 & 86.7 & $0.964(0.924,1.000)$ & 0.787 & 0.047 \\
\hline & 2 & 84.0 & 93.3 & $0.959(0.915,1.000)$ & 0.773 & 0.039 \\
\hline & 1 & 80.0 & 63.3 & $0.707(0.567,0.846)$ & 0.433 & 0.035 \\
\hline \multirow[t]{5}{*}{ PLS } & 5 & 100.0 & 100.0 & $1.000(1.000,1.000)$ & 1.000 & 0.812 \\
\hline & 4 & 100.0 & 100.0 & $1.000(1.000,1.000)$ & 1.000 & 0.791 \\
\hline & 3 & 100.0 & 100.0 & $1.000(1.000,1.000)$ & 1.000 & 0.690 \\
\hline & 2 & 92.0 & 100.0 & $0.991(0.974,1.000)$ & 0.920 & 0.582 \\
\hline & 1 & 100.0 & 90.0 & $0.956(0.895,1.000)$ & 0.900 & 0.501 \\
\hline OPLS & 1 & 100.0 & 100.0 & $1.000(1.000,1.000)$ & 1.000 & 0.853 \\
\hline
\end{tabular}

Abbreviations: $A U C$ area under the curve, $C l$ confidence interval, $P C A$ principal component analysis, $P L S$-DA partial least squares-discriminate analysis, OPLS-DA orthogonal partial least squares-discriminant analysis 


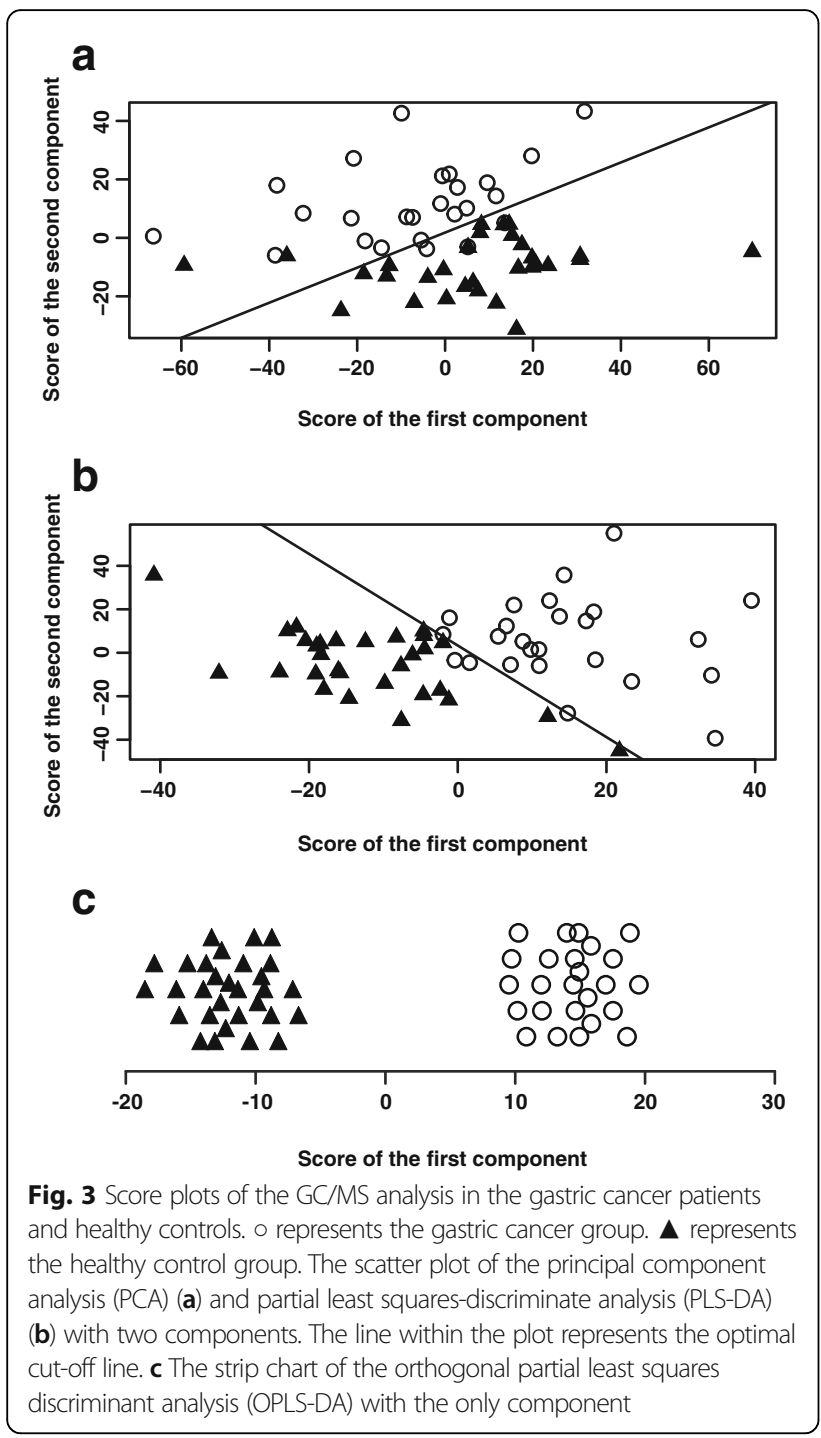

we found their diagnostic efficiencies not up to expectations. On the contrary, the combinations of microRNAs and metabolomics have the satisfactory diagnostic value constantly $[11,17]$.

MicroRNA detection has a good many advantages. Compared with long non-coding RNAs and mRNAs, microRNAs are stable and easy to amplify. The stability is reflected at room temperature and even after repeated freeze-thawing [30]. In contrast with gastroscopy, it is inexpensive and non-invasive with almost no complications. Each sample detection for six microRNAs costs approximately 28 dollars in China, which is half of the expense of gastroscopy plus biopsy. The superiority of microRNA detection would be larger in developed countries because of the fancy price of endoscopy. Nevertheless, as nucleic acids, microRNAs cannot be detected directly, and they must first be extracted and reverse transcribed.

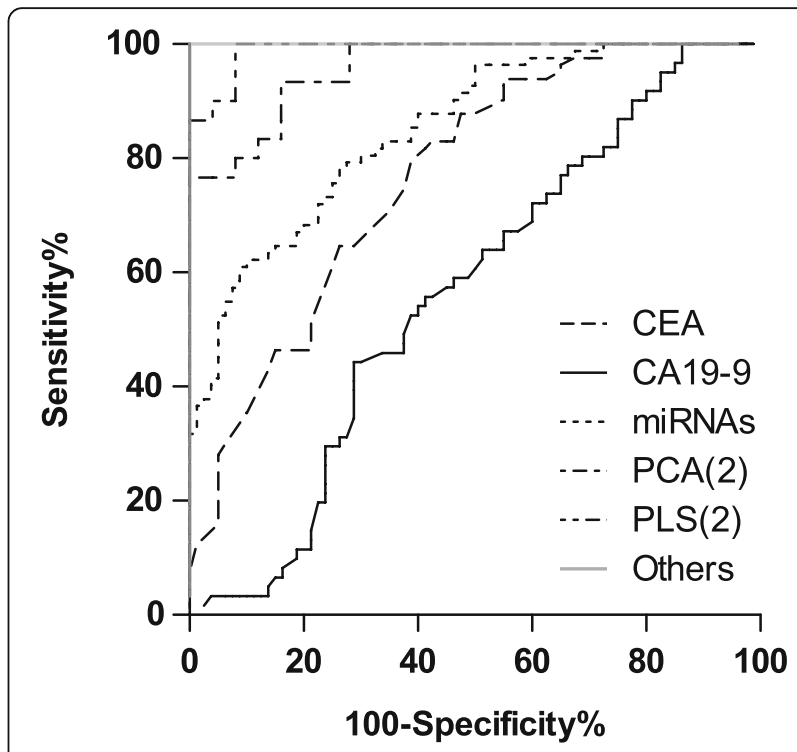

Fig. 4 Receiver operating characteristic (ROC) curves. ROC curves of the combination of miR-19a and miR-92a, the PCA and PLS-DA model with two components, CEA, CA19-9 and others for discriminating gastric cancer patients from control subjects. Others include the PCA model with seven principal components, the PLS-DA model with three to five components and the OPLS-DA model with the only component. Abbreviations: PCA, principal component analysis; PLS-DA, partial least squares-discriminate analysis; OPLS-DA, orthogonal partial least squaresdiscriminant analysis; CEA, carcinoembryonic antigen; CA19-9, carbohydrate antigen 19-9

Furthermore, fold changes and cut-off values are tremendously diverse among different studies because the choice of reference RNA, the dosage of reagents, qPCR detecting instrument and an operating process are not yet standardized. The standardization of protocol is necessary to achieve detection automation and clinical application. The expression of serum microRNAs were altered in various malignant tumors [11, 31-34]. Nevertheless, microRNA diagnostic models may be optimal in determining whether a patient has a malignant tumor. A position diagnosis can be completed through typical clinical manifestations, imaging reports and gastroscopy.

A common research routine of diagnostic test of microRNAs is to screen by the microarray in a small sample size and then validate the results by qRT-PCR in a larger sample size [35]. Other studies validated by qRT-PCR directly after screening from microRNA databases. We chose microRNAs with high diagnostic value via meta-analyses. In view of including more subjects, the selection of microRNAs are more reliable. Three of these microRNAs have potential to become independent biomarkers (AUC >0.7). It is somewhat disappointing that the combinations of microRNAs didn't increase the AUC value substantially when we attempted all probable combinations of microRNAs. The combination of miR-18a, miR-19a, miR-21, 
miR-92a and miR-199a had the AUC value at 0.867 (Table 3). However, it was not significantly different compared to the combination of miR-19a and miR-92a according to the logistic regression.

Similar to previous studies on circulating metabolomics in GC patients, endogenous metabolites, such as amino acids, organic acids, carbohydrates, fatty acids and steroids, were detected with significant differences [36-38]. These varieties suggested metabolism of tumor cells disturbed several metabolic pathways in patients. As a kind of omics technology, metabolomics show a great advantage in diagnosis of GC. It is conceivable that there are hundreds of thousands of low-molecularweight metabolites that change the concentrations in patients with malignant tumor. Our preliminary experiments even indicated that different malignant tumors could be divided by metabolomics. Besides high diagnostic value, GC/MS analysis also has the affordable price, 72.5 dollars. However, the pretreatment process is not standardized, including the choice of the internal standard and derivatization reagents, the time of each step and the operating order.

Conducting the high-throughput data, the PCA, PLSDA and OPLD-DA models remain stable when the variables are numerous and the observations are sparse. The results of our study suggest that the OPLS-DA model has the highest AUC and the PCA model ranks the last when including the same number of components. The conclusion could be explained by statistics. PLS-DA and OPLS-DA are supervisory analysis methods, while PCA is non-supervisory. Based on PLS, OPLS further separates the orthogonal variables by an orthogonal signal correction [39, 40]. Although the PCA model is the worst in the three multivariate statistical methods, we could increase the AUC by extracting more principal components. We have noticed that only significantly different metabolites, usually less than ten varieties, were fitted into the diagnostic statistical models in previous studies of metabolomics. We used all 1118 metabolites to construct the model in our study and an internal validation indicated that the models with all metabolites were more robust than those with limited metabolites [41].

Compared with new diagnostic models, CEA showed the inferior diagnostic efficiencies. CEA is better to become a biomarker to predict the recurrence actually [42]. It is interesting that there was no significant differences between GC patients and HCs for CA19-9, which was more commonly used to diagnose pancreatic cancer and colorectal cancer. The cut-off value established by Youden index or Euclidean index of ROC curve could realize more potential to a biomarker than that established by the upper bound of $95 \%$ of healthy people.

\section{Conclusions}

In conclusion, the diagnostic value of the new models is higher than that of the traditional biomarkers CEA and CA19-9. We suggest that a GC/MS analysis and a combination of microRNAs allow for the clinical application to diagnosis of GC.

\section{Additional files}

Additional file 1: Table S1. List of the included studies. (DOCX $20 \mathrm{~kb}$ )

Additional file 2: Table S2. Characteristics of the included studies. Abbreviations: GC, gastric cancer; NA, not available. (DOCX 23 kb)

Additional file 3: Figure S1. Deeks' funnel plot for the assessment of publication bias. (DOCX $186 \mathrm{~kb}$ )

Additional file 4: Table S3. MicroRNA expressions and the results of the statistical test. $Z$ value was performed when Wilcoxon-Mann-Whitney test was used in the GC patients versus the control group, and $t$ value was performed when Student's t-test was used. Abbreviations: GC, gastric cancer. (DOCX $14 \mathrm{~kb}$ )

Additional file 5: Table S4. Significantly different metabolites between the gastric cancer patients and the healthy controls. Abbreviations: VIP, variable importance in the projection; $M Z$, mass-to-charge ratio; $R T$, retention time. (DOCX $15 \mathrm{~kb}$ )

\section{Abbreviations}

APBA2: Amyloid beta precursor protein binding family A member 2; AUC: Area under the curve; BSTFA: Bis-(trimethylsilyl)-trifluoroacetamide; CA19-9: Carbohydrate antigen 19-9; CBM: Chinese Biomedical Literature Database; CEA: Carcinoembryonic antigen; Cl: Confidence interval; CT: Cycle threshold or computed tomography; GC: Gastric cancer; GC/MS: Gas chromatography/mass spectrometry; HC: Healthy control; LSINCT-5: Long stress-induced non-coding transcript 5; MMP-9: Matrix metalloproteinase-9; MZ: Mass-to-charge ratio; NCCN: National Comprehensive Cancer Network; NIST: National Institute of Standards and Technology; OPLS-DA: Orthogonal partial least squares-discriminant analysis; PCA: Principal component analysis; PLS-DA: Partial least squares-discriminate analysis; qRT-PCR: Quantitative reverse-transcription polymerase chain reaction; ROC: Receiver operating curve; RT: Retention time; S100A6: S100 calcium binding protein A6; SOX17: SRY-box 10; SPG20: Spastic paraplegia-20; SROC: Summary receiver operator characteristic; STC1: Stanniocalcin-1; TMCS: Trimethylchlorosilane; UCA1: Urothelial cancer associated 1; VIP: Variable importance in the projection

\section{Acknowledgements}

The authors would like to thank the members of Prof. Xi-Zhong Shen's laboratory for helpful discussions and critical reading of the manuscript.

\section{Funding}

This study was supported by the National Nature Science Foundation of China (No. 81000968; No. 81101540; No. 81101637; No. 81172273; No. 81272388; No. 81301820; No. 81472673), Doctoral Fund of Ministry of Education of China (20120071110058), and The National Clinical Key Special Subject of China. All these study sponsors have no roles in the study design, in the collection, analysis, and interpretation of data.

\section{Availability of data and materials}

Data are stored by the corresponding author of this paper and are available upon request.

\section{Authors' contributions}

HNL, TTL, YJT, LD and XZS conceived and designed the experiments. YJT drafted the manuscript. HNL, YJC, DYZ and LZ extracted the data of systematic review and meta-analysis. HNL, HW and YJC performed the experiments of microRNA and metabolomics. HNL and HW performed the statistical analyses. LD and XZS revised the article. All authors finished reading and approving the final manuscript of this study. 


\section{Ethics approval and consent to participate}

All samples were coded anonymously in accordance with local ethical guidelines, as stipulated by the Declaration of Helsinki with written informed consent and a protocol approved by the Ethics Review Committee of Zhongshan Hospital of Fudan University with the following reference number B2016-113R, and every patient provided written informed consent before enrollment.

\section{Competing interests}

The authors declare that they have no competing interests.

\section{Publisher's Note}

Springer Nature remains neutral with regard to jurisdictional claims in published maps and institutional affiliations.

\section{Author details}

'Department of Gastroenterology, Zhongshan Hospital of Fudan University, Room 207, Building 3, Zhongshan Hospital, Fenglin Road 180\#, Xuhui District, Shanghai, China. ${ }^{2}$ Department of Geriatrics, Zhongshan Hospital of Fudan University, 180 Fenglin Road, Shanghai 200032, China. ${ }^{3}$ Shanghai Institute of Liver Diseases, Zhongshan Hospital of Fudan University, 180 Fenglin Road, Shanghai 200032, China.

\section{Received: 9 October 2017 Accepted: 8 April 2018}

\section{Published online: 13 April 2018}

\section{References}

1. Torre LA, Bray F, Siegel RL, Ferlay J, Lortet-Tieulent J, Jemal A. Global cancer statistics, 2012. CA Cancer J Clin. 2015;65:87-108.

2. Ferlay J, Shin HR, Bray F, Forman D, Mathers C, Parkin DM. Estimates of worldwide burden of cancer in 2008: GLOBOCAN 2008. Int J Cancer. 2010; 127:2893-917.

3. Ma JL, Zhang L, Brown LM, Li JY, Shen L, Pan KF, et al. Fifteen-year effects of helicobacter pylori, garlic, and vitamin treatments on gastric cancer incidence and mortality. J Natl Cancer Inst. 2012;104:488-92.

4. Herrero R, Parsonnet J, Greenberg ER. Prevention of gastric cancer. JAMA. 2014;312:1197-8.

5. Kanda M, Kodera Y. Recent advances in the molecular diagnostics of gastric cancer. World J Gastroenterol. 2015;21:9838-52.

6. Tsujiura M, Ichikawa D, Komatsu S, Shiozaki A, Takeshita H, Kosuga T, et al. Circulating microRNAs in plasma of patients with gastric cancers. $\mathrm{Br} J$ Cancer. 2010;102:1174-9.

7. Chen JL, Tang HQ, Hu JD, Fan J, Hong J, Gu JZ. Metabolomics of gastric cancer metastasis detected by gas chromatography and mass spectrometry. World J Gastroenterol. 2010;16:5874-80.

8. Bartel DP. MicroRNAs: genomics, biogenesis, mechanism, and function. Cell. 2004;116:281-97.

9. Zeng Z, Wang J, Zhao L, Hu P, Zhang H, Tang X, et al. Potential role of microRNA-21 in the diagnosis of gastric cancer: a meta-analysis. PLoS One. 2013;8:e73278.

10. Zhou X, Ji G, Chen H, Jin W, Yin C, Zhang G. Clinical role of circulating miR223 as a novel biomarker in early diagnosis of cancer patients. Int J Clin Exp Med. 2015:8:16890-8.

11. Wang R, Wen H, Xu Y, Chen Q, Luo Y, Lin Y, et al. Circulating microRNAs as a novel class of diagnostic biomarkers in gastrointestinal tumors detection: a meta-analysis based on 42 articles. PLoS One. 2014;9:e113401.

12. Liu L, Wang S, Cao X, Liu J. Diagnostic value of circulating microRNAs for gastric cancer in Asian populations: a meta-analysis. Tumour Biol. 2014;35: 11995-2004.

13. Zhu X, Lv M, Wang H, Guan W. Identification of circulating microRNAs as novel potential biomarkers for gastric cancer detection: a systematic review and meta-analysis. Dig Dis Sci. 2014;59:911-9.

14. Bu Q, Huang Y, Yan G, Cen X, Zhao YL. Metabolomics: a revolution for novel cancer marker identification. Comb Chem High Throughput Screen. 2012;15:266-75.

15. Jonsson P, Johansson Al, Gullberg J, Trygg J, A J, Grung B, et al. Highthroughput data analysis for detecting and identifying differences between samples in GC/MS-based metabolomic analyses. Anal Chem. 2005;77:5635-42.

16. Di Lena M, Travaglio E, Altomare DF. New strategies for colorectal cancer screening. World J Gastroenterol. 2013;19:1855-60.

17. Xiao S, Zhou L. Gastric cancer: metabolic and metabolomics perspectives (review). Int J Oncol. 2017;51:5-17.
18. Liu HN, Wu H, Chen YZ, Chen YJ, Shen XZ, Liu TT. Altered molecular signature of intestinal microbiota in irritable bowel syndrome patients compared with healthy controls: a systematic review and meta-analysis. Dig Liver Dis. 2017;49:331-7.

19. Chen YJ, Zhu JM, Wu H, Fan J, Zhou J, Hu J, et al. Circulating microRNAs as a fingerprint for liver cirrhosis. PLoS One. 2013;8:e66577.

20. Wu H, Xue R, Dong L, Liu T, Deng C, Zeng H, et al. Metabolomic profiling of human urine in hepatocellular carcinoma patients using gas chromatography/ mass spectrometry. Anal Chim Acta. 2009;648:98-104.

21. Chen SZ, Yao HQ, Zhu SZ, Li QY, Guo GH, Yu J. Expression levels of matrix metalloproteinase-9 in human gastric carcinoma. Oncol Lett. 2015;9:915-9.

22. Fang Z, Tian Z, Luo K, Song H, Yi J. Clinical significance of stanniocalcin expression in tissue and serum of gastric cancer patients. Chin J Cancer Res. 2014;26:602-10.

23. Zhang J, Zhang K, Jiang X, Zhang J. S100A6 as a potential serum prognostic biomarker and therapeutic target in gastric cancer. Dig Dis Sci. 2014;59:2136-44.

24. Han J, Lv P, Yu JL, Wu YC, Zhu X, Hong LL, et al. Circulating methylated MINT2 promoter DNA is a potential poor prognostic factor in gastric cancer. Dig Dis Sci. 2014;59:1160-8.

25. Zhang H, Song Y, Xia P, Cheng Y, Guo Q, Diao D, et al. Detection of aberrant hypermethylated spastic paraplegia-20 as a potential biomarker and prognostic factor in gastric cancer. Med Oncol. 2014;31:830.

26. Balgkouranidou I, Karayiannakis A, Matthaios D, Bolanaki H, Tripsianis G, Tentes AA, et al. Assessment of SOX17 DNA methylation in cell free DNA from patients with operable gastric cancer. Association with prognostic variables and survival. Clin Chem Lab Med. 2013;51:1505-10.

27. Dong L, Qi P, Xu MD, Ni SJ, Huang D, Xu QH, et al. Circulating CUDR, LSINCT-5 and PTENP1 long noncoding RNAs in sera distinguish patients with gastric cancer from healthy controls. Int J Cancer. 2015;137:1128-35.

28. Zhou SL, Ku JW, Fan ZM, Yue WB, Du F, Zhou YF, et al. Detection of autoantibodies to a panel of tumor-associated antigens for the diagnosis values of gastric cardia adenocarcinoma. Dis Esophagus. 2015;28:371-9.

29. Werner S, Chen H, Butt J, Michel A, Knebel P, Holleczek B, et al. Evaluation of the diagnostic value of 64 simultaneously measured autoantibodies for early detection of gastric cancer. Sci Rep. 2016;6:25467.

30. Mitchell PS, Parkin RK, Kroh EM, Fritz BR, Wyman SK, Pogosova-Agadjanyan EL, et al. Circulating microRNAs as stable blood-based markers for cancer detection. Proc Natl Acad Sci U S A. 2008;105:10513-8.

31. Wang B, Zhang Q. The expression and clinical significance of circulating microRNA-21 in serum of five solid tumors. J Cancer Res Clin Oncol. 2012; 138:1659-66.

32. Zheng G, Du L, Yang X, Zhang X, Wang L, Yang Y, et al. Serum microRNA panel as biomarkers for early diagnosis of colorectal adenocarcinoma. $\mathrm{Br} J$ Cancer. 2014;111:1985-92.

33. Bhattacharya S, Steele R, Shrivastava S, Chakraborty S, Di Bisceglie AM, Ray RB. Serum miR-30e and miR-223 as novel noninvasive biomarkers for hepatocellular carcinoma. Am J Pathol. 2016;186:242-7.

34. Zhang C, Wang C, Chen X, Yang C, Li K, Wang J, et al. Expression profile of microRNAs in serum: a fingerprint for esophageal squamous cell carcinoma. Clin Chem. 2010;56:1871-9.

35. Tsai MM, Wang CS, Tsai CY, Huang HW, Chi HC, Lin YH, et al. Potential diagnostic, prognostic and therapeutic targets of MicroRNAs in human gastric Cancer. Int J Mol Sci. 2016;17:945.

36. Ikeda A, Nishiumi S, Shinohara M, Yoshie T, Hatano N, Okuno T, et al. Serum metabolomics as a novel diagnostic approach for gastrointestinal cancer. Biomed Chromatogr. 2012;26:548-58.

37. Song H, Peng JS, Dong-Sheng Y, Yang ZL, Liu HL, Zeng YK, et al. Serum metabolic profiling of human gastric cancer based on gas chromatography/ mass spectrometry. Braz J Med Biol Res. 2012;45:78-85.

38. Yang T, Luo $P$, Li Y, Hua R, Yin P, Xu G. A serum metabolomics study of gastric cancer based on pseudotargeted liquid chromatography-mass spectrometry approach. Chin J Chromatogr. 2014;32:126-32.

39. Trygg J, Wold S. Orthogonal projections to latent structures (O-PLS). J Chemom. 2002;16:119-28.

40. Nguyen DV, Rocke DM. Tumor classification by partial least squares using microarray gene expression data. Bioinformatics. 2002;18:39-50.

41. Liu H, Wu H, Chen Y, Tseng Y, Bilegsaikhan E, Dong L, et al. Serum microRNA signatures and metabolomics have high diagnostic value in hepatocellular carcinoma. Oncotarget. 2017;65:108810-24.

42. Wang W, Seeruttun SR, Fang C, Chen J, Li Y, Liu Z, et al. Prognostic significance of carcinoembryonic antigen staining in Cancer tissues of gastric Cancer patients. Ann Surg Oncol. 2016;23:1244-51. 